\title{
Analytical method for the kinematics of the multi-link guiding mechanisms used for vehicle rear axle suspension
}

\author{
Cătălin Alexandru' \\ ${ }^{1}$ Product Design, Mechatronics and Environment Department, Transilvania University of \\ Braşov, Romania \\ E-mail: calex@unitbv.ro
}

\begin{abstract}
The paper deals with an analytical method for the kinematic analysis of the multi-link guiding mechanisms used for the rear beam axles of motor vehicles. The method is based on the representation of spatial movement of the axle as a screw motion. The finite rotation of the axle around the screw axis is modelled by using rotational indicators and tensors. For solving the nonlinear system that describes the kinematic behaviour of the axle guiding mechanism, an algorithm based on the Newton-Kantorovich approach was developed. The numerical application was carried out by considering an axle guiding mechanism by five points - on five spheres (codified 5-SS)
\end{abstract}

\section{Introduction}

Generally, the spatial guiding of the bodies can be achieved in two ways: by guiding a characteristic point of the body and orienting it with respect to the considered point (solution that is usually used in industrial robots), or by guiding several points of the body, the latest solution being also used for guiding the rear beam axles of the motor vehicles in relative movement to the chassis. The arrangement of the guiding points on the rear axle is made taking into account the kinematic requirements (regarding the accomplishment of the necessary movements, namely the vertical displacement of the axle and its rotation around the longitudinal axis of the car), as well as the dynamic ones (taking over the contact forces from the wheels). The structural systematization of the axle guiding mechanisms according to the possible guiding cases was depicted in [3].

In addition to the necessary specified motions of the rear axle, other additional linear and angular displacements occur during the operation of the guiding mechanism, as follows: rotations of the axle around the transversal and vertical axes, displacements of the axle centre along the transversal and longitudinal axes. These movements are undesirable and they have the effect of changing the direction of travel of the car and losing stability, changes in wheelbase and wheel track (with additional wear and consumption), increased rolling resistance, and additional stresses in the suspension system. Due to these negative effects, all the additional movements must be cancelled or minimized, as far as possible. The purpose of the kinematic analysis is to establish the functions that describe the stated movements, and to calculate their variations during the operation of the axle guiding mechanism.

Considering the specific features, the kinematic analysis of the axle guiding mechanisms is based on simplified models in which the chassis is fixed connected to ground (thus being the reference part of the mechanism), and the force generating elements (such as springs, dampers, tires, bumpers, anti-roll bars) are not taken into account. The connections of the guiding links/arms to the adjacent parts (axle and chassis) are commonly modelled by spherical joints [3, 12-14, 16]. 
In the scientific literature, the kinematic analysis of the axle guiding mechanisms is achieved in two ways: by developing analytical methods, which consist of formulating and solving the equation systems that describe the kinematic behaviour [3, 5, 7, 8, 15]; by using commercial MBS (Multi-Body Systems) software solutions, which automatically formulate and solve the movement equations $[4,9,17,18]$. The use of MBS software brings important benefits for various types of applications, such as those presented in $[1,2,6,10]$, but their high cost is still a major impediment.

This paper deals with the development of a numerical method for the kinematic analysis of the axle guiding mechanisms. The method is a general one, which can be applied for all types of rear axle guiding mechanisms (at least the frequently used ones).

\section{Problem formulation}

The numerical method proposed in this work is based on the modelling of the spatial movement (position and orientation) of the rear axle as a screw movement [11], considering the rotation around the screw axis $u$ (i.e. the angle $\beta$ ) and the translation along this axis (i.e. the displacement b), as shown in Figure 1.

The axle position, with reference to the origin $\mathrm{O}$ of the global coordinate system, is determined by the position vector $r_{u}$. In order to define the axle spatial movement, the position vector, velocity and acceleration of a certain point $\mathrm{M}$ on the axle must be known. The global position of this point is defined in the following way

$$
\bar{r}_{M}=\bar{r}_{G}+\bar{q},
$$

where $\mathrm{r}_{\mathrm{G}}$ is the global position vector of a reference point $\mathrm{G}$, while $\mathrm{q}$ is the local position vector of $\mathrm{M}$ relative to $\mathrm{G}$.
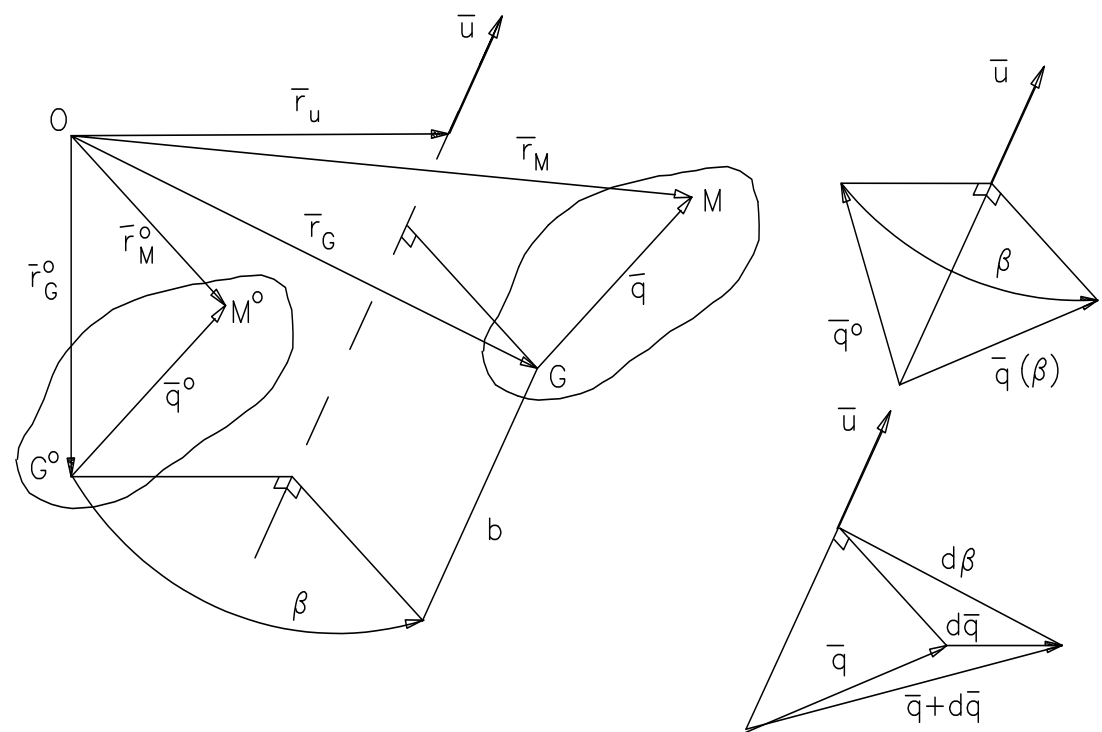

Figure 1. The modelling of the screw movement of the rear axle.

In figure 1 , the vector q defines the orientation of the rear axle, and it results by the rotation around the screw axis from the initial configuration $\mathrm{q}^{0}$, while $\mathrm{r}_{\mathrm{G}}$ is used to model the position of the axle. The scientific literature reveals several methods for modelling the orientation of the vector $\mathrm{q}$ around the screw axis u, such as Euler angles, Bryant angles, Kardan angles, director cosines, rotational indicators and tensors, and others. In what follows, the method based on rotational indicators and tensors [11] will be used. 
The rotational indicator $\beta=(\mathrm{u}, \beta)$ contains the three components of vector $\mathrm{u}$ and the corresponding revolute angle $\beta$. The finite rotation of the vector $q$ (having the initial configuration $q^{0}$ ) is obtained by integrating the differential rotation around the instant axis of angle $\mathrm{d} \beta$,

$$
\begin{gathered}
d \bar{q}=\bar{u} \cdot d \beta \times \bar{q} \rightarrow \bar{q}(\bar{\beta})=T(\bar{\beta}) \cdot \bar{q}^{-0}, \\
\mathrm{~T}(\beta)=\mathrm{I}+\sin \beta \cdot \mathrm{U}+(1-\cos \beta) \cdot \mathrm{U}^{2}, \quad U=\left[\begin{array}{ccc}
0 & -u_{z} & u_{y} \\
u_{z} & 0 & -u_{x} \\
-u_{y} & u_{x} & 0
\end{array}\right],
\end{gathered}
$$

where $T(\beta)$ is the rotational tensor that describes the finite rotation of the vector $q$ around the screw axis $q$ (of angle $\beta$ ), I - the unit tensor, and $\mathrm{U}$ - the anti-symmetric tensor.

Of the four parameters of the rotational indicator, due to the condition $|\mathrm{u}|=1$, only three are independent. The passing to three parameters is done by introducing the vector $\mathrm{w}$ of the same direction with $\mathrm{u}$,

$$
\bar{w}=\bar{u} \cdot \operatorname{tg} \frac{\beta}{2},
$$

whose components are the Rodrigues parameters of the finite rotation (the three-parametric representation of the finite rotations). The rotational tensor $\mathrm{T}(\beta)$ will be:

$$
T(\bar{\beta})=T(\bar{w})=I+2 \cdot \frac{W+W^{2}}{1+\bar{w} \cdot \bar{w}},
$$

where:

$$
W=\left[\begin{array}{ccc}
0 & -w_{z} & w_{y} \\
w_{z} & 0 & -w_{z} \\
-w_{y} & w_{x} & 0
\end{array}\right]
$$

The analytical representation of the screw movement of the point $\mathrm{M}$ is given by:

$$
\bar{r}_{M}\left(\bar{r}_{G}, \bar{w}\right)=\bar{r}_{G}+T(\bar{w}) \cdot \bar{q}^{-},
$$

where the six components of the vectors $\mathrm{w}$ and $\mathrm{r}_{\mathrm{G}}$ are independent motion parameters.

\section{Case study}

The application for this paper corresponds to an axle guiding mechanism by five points - on five spheres (5-SS), which is shown in Figure 2. The mechanism, which has a single degree of mobility, contains contains four longitudinal bars, up-down arranged relative to the axle axis in pairs of two $\left(1_{\mathrm{s}}-1_{\mathrm{d}}, 3_{\mathrm{s}}-3_{\mathrm{d}}\right)$, while the fifth bar (4), which is called Panhard bar, is transversally disposed.

The geometrical model of this guiding mechanism is defined by the following parameters: the global coordinates of the joints $\mathrm{M}_{0 \mathrm{i}}$ of the guiding bars to chassis, in the global coordinate system OXY; the local coordinates of the joints $\mathrm{M}_{\mathrm{i}}$ of the guiding bars to axle, in the axle reference frame $\mathrm{PX}_{\mathrm{P}} \mathrm{Y}_{\mathrm{P}} \mathrm{Z}_{\mathrm{P}}$; the static position of the axle, in OXYZ; the lengths of the guiding bars; the wheel radius and the wheel track. 
ISSN: 2668-0416

Thoth Publishing House

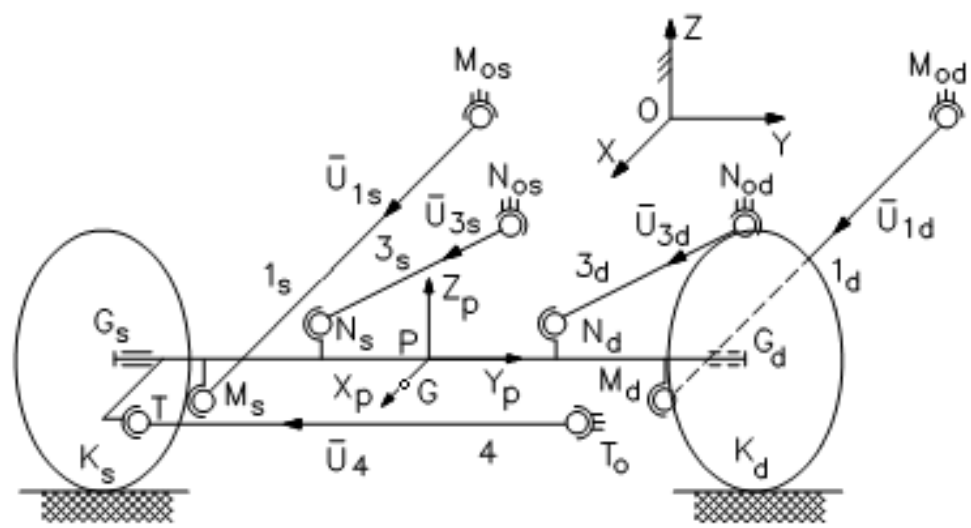

Figure 2. The axle guiding mechanism of type 5SS.

The center of the left wheel $\left(G_{s}\right)$ is considered as reference point (corresponding to $G$ in equation (1)), from equations (7) resulting:

$$
F_{i}=\left[{ }^{r} G s+T(\bar{w}) \cdot\left(\bar{r}_{M i}-\bar{r}_{G s}\right)_{0}-\bar{r}_{M O i}\right]^{2}-l_{i}^{2}=0,
$$

where $\mathrm{i}=1 \ldots . .5 ; \mathrm{M}_{\mathrm{i}} \in\left[\mathrm{M}_{\mathrm{s}}, \mathrm{M}_{\mathrm{d}}, \mathrm{N}_{\mathrm{s}}, \mathrm{N}_{\mathrm{d}}, \mathrm{T}\right] ; \mathrm{M}_{0 \mathrm{i}} \in\left[\mathrm{M}_{0 \mathrm{~s}}, \mathrm{M}_{0 \mathrm{~d}}, \mathrm{~N}_{0 \mathrm{~s}}, \mathrm{~N}_{0 \mathrm{~d}}, \mathrm{~T}_{0}\right]$.

The rotational tensor $\mathrm{T}(\mathrm{w})$ is defined by the following components:

$$
\begin{aligned}
& t_{11}=1-A \cdot\left(w_{y}^{2}+w_{z}^{2}\right), t_{12}=A \cdot\left(-w_{z}+w_{x} \cdot w_{y}\right), t_{13}=A \cdot\left(w_{y}+w_{x} \cdot w_{z}\right), \\
& t_{21}=A \cdot\left(w_{z}+w_{x} \cdot w_{y}\right), t_{22}=1-A \cdot\left(w_{x}^{2}+w_{z}^{2}\right), t_{23}=A \cdot\left(-w_{x}+w_{y} \cdot w_{z}\right), \\
& t_{31}=A \cdot\left(-w_{y}+w_{x} \cdot w_{z}\right), t_{32}=A \cdot\left(w_{x}+w_{y} \cdot w_{z}\right), t_{33}=1-A \cdot\left(w_{x}^{2}+w_{y}^{2}\right),
\end{aligned}
$$

where

$$
A=\frac{2}{1+\bar{w} \cdot \bar{w}}=\frac{2}{1+w_{x}^{2}+w_{y}^{2}+w_{z}^{2}} .
$$

Equation system (8) can be rewritten in the following form:

$$
\begin{aligned}
& F_{i}=\left[X_{G s}-X_{M 0 i}+t_{11}\left(X_{M i}-X_{G s}\right)_{0}+t_{12}\left(Y_{M i}-Y_{G s}\right)_{0}+t_{13}\left(Z_{M i}-Z_{G s}\right)_{0}\right]^{2}+ \\
& +\left[Y_{G s}-Y_{M 0 i}+t_{21}\left(X_{M i}-X_{G s}\right)_{0}+t_{22}\left(Y_{M i}-Y_{G s}\right)_{0}+t_{23}\left(Z_{M i}-Z_{G s}\right)_{0}\right]^{2}+ \\
& +\left[Z_{G s}-Z_{M 0 i}+t_{31}\left(X_{M i}-X_{G s}\right)_{0}+t_{32}\left(Y_{M i}-Y_{G s}\right)_{0}+t_{33}\left(Z_{M i}-Z_{G s}\right)_{0}\right]^{2}-l_{i}^{2}=0 .
\end{aligned}
$$

The vertical displacement of the left wheel centre $\left(\mathrm{Z}_{\mathrm{Gs}}\right)$ is selected as independent kinematic parameter, while the unknowns are $\mathrm{X}_{\mathrm{Gs}}, \mathrm{Y}_{\mathrm{Gs}}, \mathrm{w}_{\mathrm{x}}, \mathrm{w}_{\mathrm{y}}$, and $\mathrm{w}_{\mathrm{z}}$. In the initial position, the global coordinates of the guiding points and those of the wheel centre can be established depending on the stationary position (vehicle in rest) of the axle in the global reference frame OXYZ and the corresponding local coordinates in the axle reference frame $\mathrm{PX}_{\mathrm{P}} \mathrm{Y}_{\mathrm{P}} \mathrm{Z}_{\mathrm{P}}$, as follows:

$$
\begin{aligned}
& X_{M i}=X_{P}^{0}+X_{M i(P)}, Y_{M i}=Y_{P}^{0}+Y_{M i(P)}, Z_{M i}=Z_{P}^{0}+Z_{M i(P)}, \\
& X_{G s}=X_{P}^{0}+X_{G s(P)}, Y_{G s}=Y_{P}^{0}+Y_{G s(P)}, Z_{G s}=Z_{P}^{0}+Z_{G s(P)} .
\end{aligned}
$$


The solving the non-linear system (11) was carried out by using the Newton-Kantorovich method. The initial solution of the non-linear system corresponds to the stationary position of the vehicle, while for a current position of the guiding mechanism, the initial solution is that obtained in the previous position, and in this way the spatial positioning of the guiding mechanism (in terms of parametric vectors of the axle finite movement, namely $\mathrm{r}_{\mathrm{Gs}}$ and $\mathrm{w}$ ) can be determined for the whole vertical travel of the axle, transposed by the variation field of the independent parameter $\left(\mathrm{Z}_{\mathrm{Gs}}\right)$.

The global coordinates of the guiding points $\mathrm{M}_{\mathrm{i}}$ are then determined based on equations (7):

$$
\left[\begin{array}{c}
X_{M i} \\
Y_{M i} \\
Z_{M i}
\end{array}\right]=\left[\begin{array}{c}
X_{G s} \\
Y_{G s} \\
Z_{G s}
\end{array}\right]+T(\beta) \cdot\left[\begin{array}{c}
X_{M i}-X_{G s} \\
Y_{M i}-Y_{G s} \\
Z_{M i}-Z_{G s}
\end{array}\right]_{O}
$$

Given that

$$
u_{x}=\frac{w_{x}}{\operatorname{tg} \frac{\beta}{2}}, u_{y}=\frac{w_{y}}{\operatorname{tg} \frac{\beta}{2}}, u_{z}=\frac{w_{z}}{\operatorname{tg} \frac{\beta}{2}},
$$

there is obtained

$$
w_{x}^{2}+w_{y}^{2}+w_{z}^{2}=\left(u_{x}^{2}+u_{y}^{2}+u_{z}^{2}\right) \cdot \operatorname{tg}^{2} \frac{\beta}{2}, \beta=2 \operatorname{arctg}\left(\mathrm{w}_{\mathrm{x}}{ }^{2}+\mathrm{w}_{\mathrm{y}}{ }^{2}+\mathrm{w}_{\mathrm{z}}{ }^{2}\right),
$$

where the rotational tensor $\mathrm{T}(\beta)$ has the following componenents:

$$
\begin{gathered}
t_{11}{ }^{\beta}=1-(1-\cos \beta) \cdot\left(u_{y}{ }^{2}+u_{z}{ }^{2}\right), t_{12}{ }^{b}=-u_{z} \cdot \sin \beta+(1-\cos \beta) \cdot u_{x} \cdot u_{y}, \\
t_{13}{ }^{\beta}=u_{y} \cdot \sin \beta+(1-\cos \beta) \cdot u_{x} \cdot u_{z} \\
t_{21}{ }^{\beta}=u_{z} \cdot \sin \beta+(1-\cos \beta) \cdot u_{x} \cdot u_{y}, t_{22}{ }^{\beta}=1-(1-\cos \beta) \cdot\left(u_{x}{ }^{2}+u_{z}{ }^{2}\right), \\
t_{23}{ }^{\beta}=-u_{x} \cdot \sin \beta+(1-\cos \beta) \cdot u_{y} \cdot u_{z} \\
t_{31}{ }^{\beta}=-u_{y} \cdot \sin \beta+(1-\cos \beta) \cdot u_{x} \cdot u_{z} t_{32}{ }^{\beta}=u_{x} \cdot \sin \beta+(1-\cos \beta) \cdot u_{y} \cdot u_{z}, \\
t_{33}{ }^{\beta}=1-(1-\cos \beta) \cdot\left(u_{x}{ }^{2}+u_{y}{ }^{2}\right) .
\end{gathered}
$$

For computing the coordinates for any other point of interest on axle (e.g. the axle center P), equations of form (13) can be used.

Afterwards, the kinematic functions that describe the behavior of the axle guiding mechanism can be determined, namely the linear displacements of the axle center along the longitudinal $\left(\Delta \mathrm{X}_{\mathrm{P}}\right)$, transversal $\left(\Delta \mathrm{Y}_{\mathrm{P}}\right)$ and vertical $\left(\Delta \mathrm{Z}_{\mathrm{P}}\right)$ axes (as shown in Figure 3), and respectively the rotations of the axle around the longitudinal $\left(\eta_{\mathrm{x}}\right)$, transversal $\left(\eta_{\mathrm{y}}\right)$ and vertical $\left(\eta_{\mathrm{z}}\right)$ axes (Figure 4$)$, as follows:

- displacements of the axle center:

$$
\Delta X_{P}=X_{P}-X_{P}^{0}, \Delta Y_{P}=Y_{P}-Y_{P}^{0}, \Delta Z_{P}=Z_{P}-Z_{P}^{0}
$$

- axle roll rotation:

$$
\eta_{\mathrm{X}}=\operatorname{arctg} \frac{\mathrm{Z}_{\mathrm{Gd}}-\mathrm{Z}_{\mathrm{Gs}}}{\mathrm{Y}_{\mathrm{Gd}}-\mathrm{Y}_{\mathrm{Gs}}}
$$

- axle rotation around its own axle (where $\mathrm{G}$ is a point located on the local axis $\mathrm{X}_{\mathrm{P}}$ of the axle reference frame $\mathrm{PX}_{\mathrm{P}} \mathrm{Y}_{\mathrm{P}} \mathrm{Z}_{\mathrm{P}}$ - see Figure 2): 


$$
\eta_{Y}=\operatorname{asin} \frac{\left(Z_{G}-Z_{G}^{0}\right)-\left(Z_{P}-Z_{P}^{0}\right)}{\left|X_{G(P)}\right|} ;
$$

- axle pivoting rotation:

$$
\eta_{\mathrm{Z}}=\operatorname{arctg} \frac{X_{\mathrm{Gd}}-X_{\mathrm{Gs}}}{Y_{\mathrm{Gd}}-Y_{\mathrm{Gs}}} .
$$

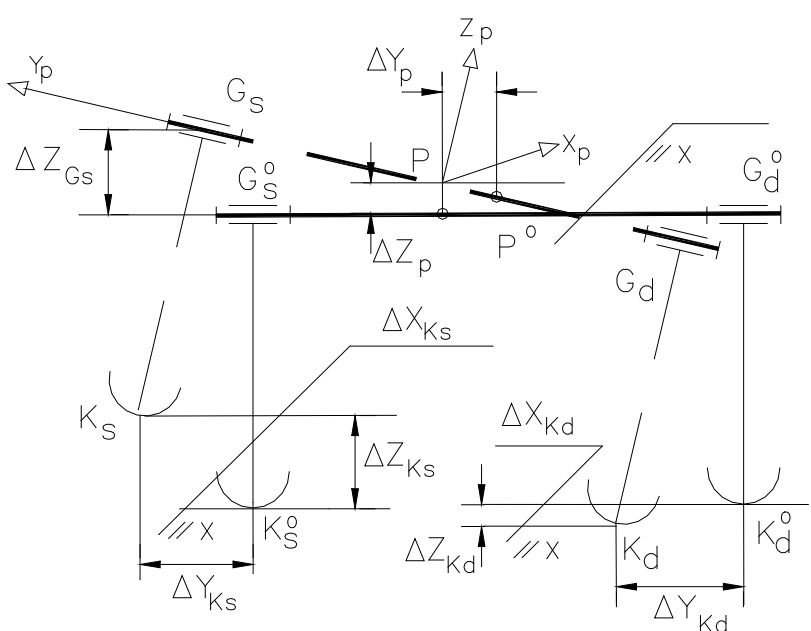

Figure 3. The linear displacements of the rear axle.

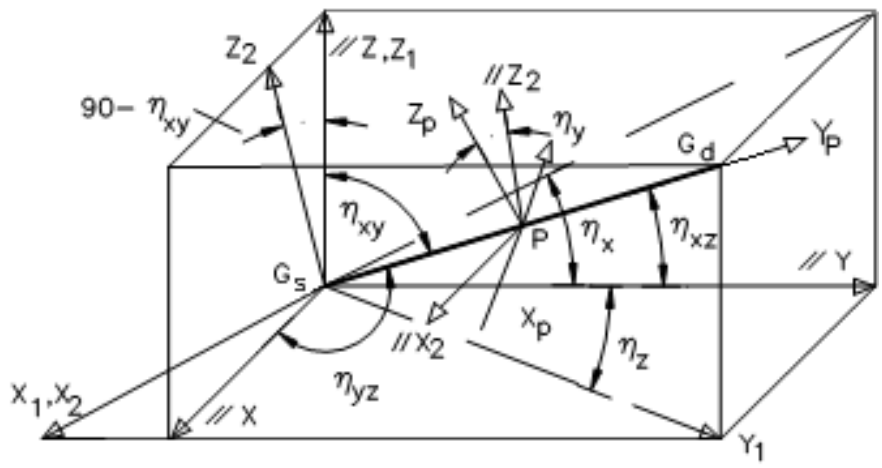

Figure 4. The orientation angles of the rear axle.

\section{Results and conclusions}

The method presented above was algorithmized and transposed on computer by using the programming software environment Borland Delphi (based on Object Pascal). The numerical simulation for this work was carried out by considering the following values of the input data for the kinematic analysis (in correlation with the notations in Figure 2):

- the global coordinates of the joints to chassis: $\mathrm{M}_{0 \mathrm{~s}}(1624.0,-457.0,79.0), \mathrm{M}_{0 \mathrm{~d}}(1624.0,457.0$, $79.0), \mathrm{N}_{0 \mathrm{~s}}(1885.0,-255.0,252.0), \mathrm{N}_{0 \mathrm{~d}}(1885.0,255.0,252.0), \mathrm{T}_{0}(2538.0,457.5,139.0)[\mathrm{mm}]$;

- the global coordinates of the joints to axle: $\mathrm{M}_{\mathrm{s}(\mathrm{P})}(-58.0,-457.0,-58.0), \mathrm{M}_{\mathrm{d}(\mathrm{P})}(-58.0,457.0,-58.0)$, $\mathrm{N}_{\mathrm{s}(\mathrm{P})}(31.0,-205.0,114.0), \mathrm{N}_{\mathrm{d}(\mathrm{P})}(31.0,205.0,114.0), \mathrm{T}(101.5,-457.0,0.0)[\mathrm{mm}] ;$

- the static/initial position of the axle center: $\mathrm{P}^{0}(2400.0,0.0,145.0)[\mathrm{mm}]$;

- the lengths of the guiding bars: $1_{1 \mathrm{~s}, \mathrm{~d}}=718.04,1_{3 \mathrm{~s}, \mathrm{~d}}=548.33,1_{4}=915.25[\mathrm{~mm}]$. 
By the kinematic analysis, the results that describe the behavior of the axle guiding mechanism have been obtained. The diagrams in Figures 5 and 6 show the displacements of the axle center along the global reference frame axes, as well as the three rotations that determine the spatial orientation of the axle/guiding mechanism. The imposed variation field for the independent kinematic parameter is $\mathrm{Z}_{\mathrm{Gs}} \in[-$ $80,80] \mathrm{mm}$, thus simulating the up/down travel of the wheel/axle in a passing over bumps regime.

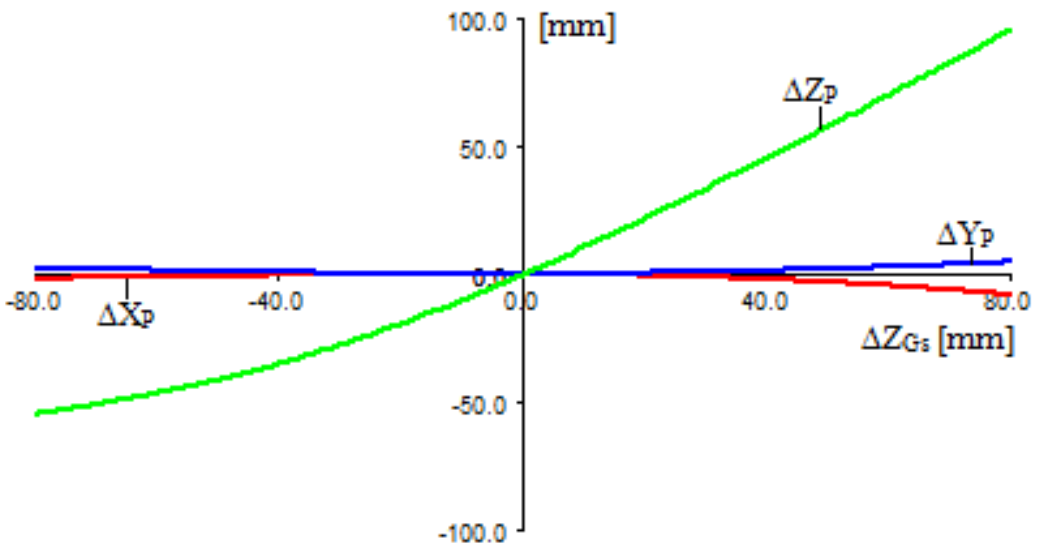

Figure 5. The linear displacements of the axle centre.

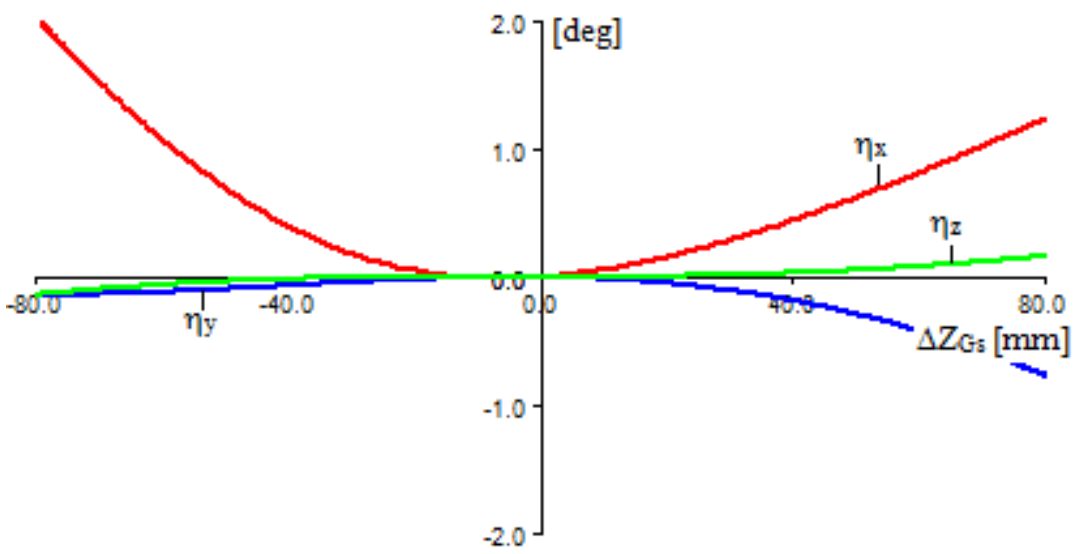

Figure 6. The orientation angles of the axle.

Based on the study presented in this paper, the following conclusions can be drawn: the proposed method uses geometric parameters of the axle guiding mechanism that can be read directly from the execution and overall drawings; by choosing as an independent parameter the vertical movement of the wheel center, there is a good correlation with the actual load on the car; the coordinates of any point on the axle are easy to calculate.

By reference to other methods from literature, the numerical algorithm proposed in this work brings several important benefits for the kinematic analysis of the axle guiding mechanisms, such as the high degree of applicability, the fast convergence of the non-linear system used to model the kinematic behavior, and the possibility to be adapted for the multi-link guiding mechanisms of the front wheels (with independent suspension).

The method here presented can be integrated / implemented in a more complex numerical algorithm for establishing the static equilibrium position of the axle suspension system (by taking into account the elastic and damping elements, as well as the system of external forces applied to the car), this being one of the directions for further research in the field. 


\section{References}

[1] Alexandru C, Comşiț M 2007 Virtual prototyping of the solar tracking systems Renewable Energy and Power Quality Journal 1(5) pp 105-110

[2] Alexandru C 2009 Software platform for analyzing and optimizing the mechanical systems Proceedings of the 10th IFToMM International Symposium on Science of Mechanisms and Machines - SYROM pp 665-677

[3] Alexandru, C 2009 The kinematic optimization of the multi-link suspension mechanism used for rear axle of the motor vehicle Proceedings of the Romanian Academy - Series A 10(3) pp 244253

[4] Alexandru C 2012 Optimal design of the mechanical systems using parametric technique \& MBS (Multi-Body Systems) software Advanced Materials Research 463-464 pp 1129-1132

[5] Alexandru C 2020 Method for the kinematic analysis of the vehicle axle guiding mechanisms. International Journal of Modeling and Optimization 10(4) pp 126-131

[6] Alexandru P, Macaveiu D, Alexandru C 2012 Design and simulation of a steering gearbox with variable transmission ratio Proceedings of the Institution of Mechanical Engineers, Part C: Journal of Mechanical Engineering Science 226(10) pp 2538-2548

[7] Attia H 2013 Kinematic analysis of the multi-link five-point suspension system in point coordinates Journal of Mechanical Science and Technology 17(8) pp 1133-1139

[8] Balike K, Rakheja S, Stiharu I 2008 Kinematic analysis and parameter sensitivity to hard points of five-link rear suspension mechanism of passenger car Proceedings of the Design Engineering Technical Conference pp 755-764

[9] Ceccarelli M 2009 Challenges for mechanism design Proceedings of the 10th IFToMM International Symposium on Science of Mechanisms and Machines - SYROM pp 1-13

[10] Enescu M, Alexandru C 2012 Modeling and simulation of a 6 DOF robot Advanced Materials Research 463-464 pp 1116-1119

[11] Hiller M, Woernle C 1985 Kinematical analysis of a five point wheel suspension ATZ 87(2) pp 59-64

[12] Knapczyk J, Maniowski M 2002 Selected effects of bushings characteristics on five-link suspension elastokinematics Mobility and Vehicle Mechanics 3(2) pp 107-121

[13] Knapczyk J, Maniowski M 2006 Elastokinematic modeling and study of five-rod suspension with subframe Mechanism and Machine Theory 41(9) pp 1031-1047

[14] Rocca E, Russo R 2002 A feasibility study on elastokinematic parameter identification for a multilink suspension Journal of Automobile Engineering 216(2) pp 153-160

[15] Simionescu P A, Beale D 2002 Synthesis and analysis of the five-link rear suspension system used in automobile Mechanism and Machine Theory 37(9) pp 815-832

[16] Tică M, Dobre G, Mateescu V 2014 Influence of compliance for an elastokinematic model of a proposed rear suspension International Journal of Automotive Technology 15(6) pp 885-891

[17] Țoțu V, Alexandru C 2013 Multi-criteria kinematic optimization of a front multi-link suspension mechanism using DOE screening and regression model Applied Mechanics and Materials 332 pp 351-356

[18] Țoțu V, Alexandru C 2014 Optimal design of the front suspension mechanism used for a race car Mechanisms and Machine Science 18 pp 243-253 